\title{
Automatic Traffic Rules Violation Detection and Number Plate Recognition System for Bangladesh
}

\author{
Raian Shahrear, Md. Anisur Rahman, Atif Islam, Chamak Dey, Md. Saniat Rahman Zishan
}

\begin{abstract}
The traffic controlling system in Bangladesh has not been updated enough with respect to fast improving technology. As a result, traffic rules violation detection and identification of the vehicle has become more difficult as the number of vehicles is increasing day by day. Moreover, controlling traffic is still manual. To solve this problem, the traffic controlling system can be digitalized by a system that consists of two major parts which are traffic rules violation detection and number plate recognition. In this research, these processes are done automatically which is based on machine learning, deep learning, and computer vision technology. Before starting this process, an object on the road is identified through the YOLOv3 algorithm. By using the OpenCV algorithm, traffic rules violation is detected and the vehicle that violated these rules is identified. To recognize the number plate of the vehicle, image acquisition, edge detection, segmentation of characters is done sequentially by using Convolution Neural Network (CNN) in MATLAB background. Among the traffic rules, the following traffic signal is implemented in this research.
\end{abstract}

Index Terms - YOLOv3, Darknet, OpenCV, Object detection, Traffic rule, Violation detection, ANPR.

\section{INTRODUCTION}

$\mathrm{T}$ RAFFIC controlling and navigation system was adopted when the transportation system was occupied by the engine-driven vehicle. Several traffic laws were also made to decrease the number of road accidents, traffic jams and so on. The first manually controlled electric traffic lights were

Raian Shahrear completed B.Sc. in Electrical and Electronic Engineering (EEE) from American International University-Bangladesh, Dhaka, Bangladesh.

Email: raianshahrearbd@gmail.com

Md. Anisur Rahman completed B.Sc. in Electrical and Electronic Engineering (EEE) from American International University-Bangladesh, Dhaka, Bangladesh.

Email: anisur_rahman@ieee.org

Atif Islam completed B.Sc. in Electrical and Electronic Engineering (EEE) from American International University-Bangladesh, Dhaka, Bangladesh. Email: ahmadatif49@gmail.com

Chamak Dey completed B.Sc. in Electrical and Electronic Engineering (EEE) from American International University-Bangladesh, Dhaka, Bangladesh.

Email: chamakdey163@gmail.com

Md. Saniat Rahman Zishan is an Associate Professor at the Department of Electrical and Electronic Engineering (EEE) \& Computer Engineering (CoE) and Head of Department of $\mathrm{CoE}$ of American International UniversityBangladesh, Dhaka, Bangladesh.

Email: saniat@aiub.edu installed in Piccadilly, London, in 1925 [1]. The system is controlled by the traffic controlling agency. The first vehicle register plate using law was passed in New York in Apr. 25, 1901 [2]. The vehicle number plate contains information about the vehicle owner's district, metropolitan, type of the car. For manual traffic controlling, when a vehicle violates traffic rules the traffic police check the vehicle's number by the manual process. As the traffic controlling process evolved, the manual process replaced by an automated checking and imposing fine which is adopted by a few first world countries. An automated system like image recognition and processing technology is used there. In a developing country like Bangladesh, the number of vehicles is increasing in recent time but the traffic controlling process is not updated [3]. From some of the previous research, it is seen that Vehicle Number Plate Recognition (VNPR) in traffic monitoring systems is controlling traffic volume, generating tickets for the vehicle with no human control, vehicle tracking and vehicle policing and vehicle security [4]. From further research, vehicle tracking using a number plate recognition system is found. It is necessary to capture the number plate of the vehicle and use this captured number to track the path of the vehicle. To track they used to extract numbers from the captured image, using JAVA, Optical Character Recognition (OCR) or MATLAB libraries. Some of them used python or $\mathrm{C}++$ along with OpenCV. There is seen that the algorithm of number plate recognition was almost the same [5]. But Automated Number Plate Recognition (ANPR) is not entirely implemented, human hand used to stop the violated vehicle and send the plate number to the processing unit. Researchers are still now working on it. Among the algorithms, Python and OpenCV are the best options to automate this system. Dubai's Roads and Transport Authority (RTA) launch a smart traffic controlling system where a radar including camera, sensors, transmitterreceiver, data server and few electronic devices have been used to recognize number plate, detect speed and wrong Uturn, unauthorized vehicle, and after generating fine with an attached violated picture. This research was almost similar to the part of the available technology where two cameras were used, one was at a high angle to detect the violation and another one was at eye angle to capture an image of a vehicle. The difference, however, was that this was not made in realtime. So, the future plane is to make it possible [6].

The main objective of this research is to recognize the number plate of a violated vehicle by following some steps, like edge detection, symmetry detection, binarization, 
projection, moving average filters, extreme detection, and OCR in the ANPR system. In computer vision and image processing edge detection method is widely used. Among the aims of edge detection, the main target is to detect and extract the feature. Identifying of edges in an image has many processes which are shown in the publication. A 3-edged detection algorithm is used like Horizontal edge, Vertical edge, and Canny edge detection in this preferred action [7]. Image binarization is the process of converting a black and white image to a 256 gray level [8]. Before the OCR binarization is widely used as pre-processing. The simplest way to convert a grayscale image to a binary image is to specify the full border value, classify all pixels like white and classify the rest of pixels with values like higher than black [9]. Symmetry is an important feature of artificial products. Most of the vehicles are symmetrical along the vertical axis at the rear or front. This property is used to identify the vehicles from an image and this is one of the main parts of the operation of number plate identification. Gray leveling is not functional for symmetrical patterns. This is because of the same area and strong redistribution can have a higher level of symmetry. These powerful reapplications create deformity in symmetry of vehicles, during a similar field and the patterns of framework introduce the most interconnected symmetry [10]. Moving average filter is familiar as playback average. This is used to facilitate multiple datasets, decreasing the impact of unsystematic changes in the data. Its primary target is to eliminate high-frequency elements and short term oscillations from the data set. A moving average is usually applied as a lower passage in digital signal processing [11]. The first process of text recognition is to check the text of the document and convert the characters into code that is used to process the data. OCR is sometimes referred to as text recognition [12]. The behavior of the processing unit or neuron is particularly dependent on activation operations. Activation operations can be expressed in 3 categories such as linear, threshold, and segment. To get maximum efficiency value in this research YOLOv3 network model is used and this is the improved version of the YOLO network [13]. In this research, object detection is going to detect through the YOLOv3 algorithm. To detect the traffic rules violation and number plate recognition, OpenCV is being used in this research.

\section{Architecture Of the System}

\section{A. System Block Diagram}

Surveillance through the camera is not new to the recent trend of the world but applying different algorithms for different recognition is new as a real-time recognition system is applying on real-time video monitoring surveillance cameras. This system will create the easiest solution to detect every vehicle. Figure 1, shows the process of the proposed method. In the detection process, software YOLOv3 is used to analyze the data from the given dataset.

At first, the videos have been collected and converted into images frame by frame. Then the frame of images from the real-time video feed will be matched to the dataset [14]. The whole system was implemented using the Python programming language. For object detection, YOLOv3 was used and OpenCV was used for violation detection and character recognition. To recognize vehicles on the road object detection system was implemented using the YOLOv3 algorithm. For this purpose, the Linux operating system was chosen since it has its default trained data to detect various object including vehicles.

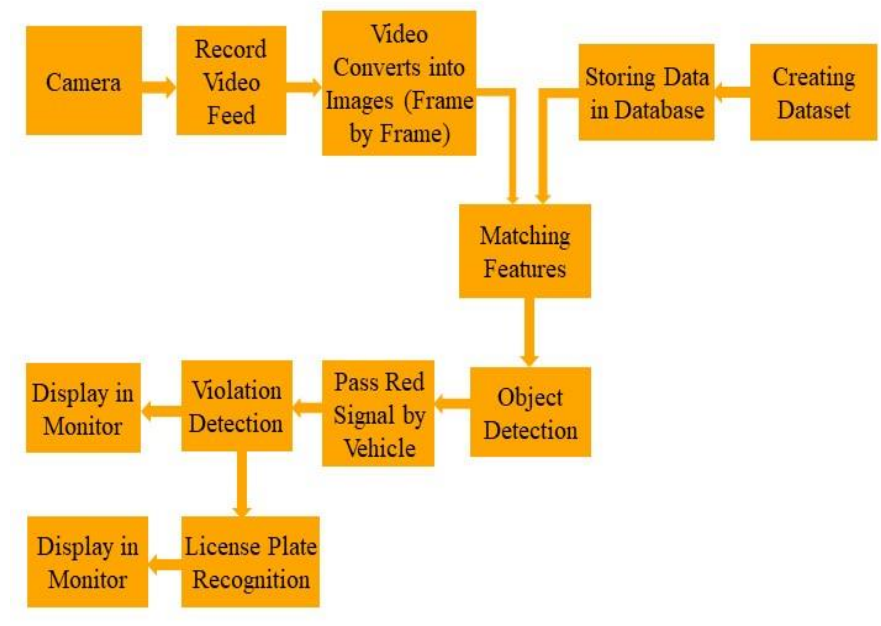

Fig. 1. Block diagram of the system

B. Flow Chart for overall system

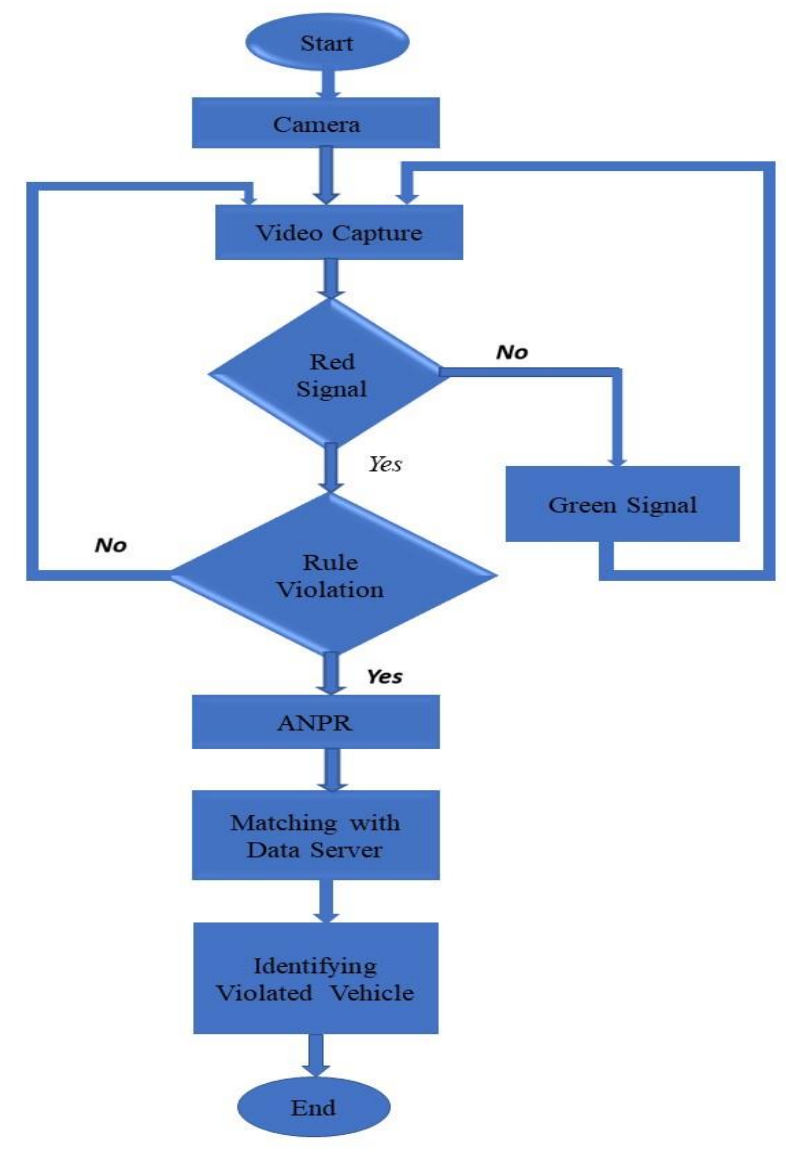

Fig. 2. System Flow Chart for the overall system 
In figure 2, the flow chart of the overall system is shown. The systematizing of automatic traffic rule violation detection and number plate recognition system capitally has two main parts (i) Traffic rules violation detection (ii) Number plate recognition. The first video of vehicles is captured through a zoom lens camera at a perfect place. After that, Red and Green lines are drawn on the video frame by using the code of python platform where these are indicated as red and green lights respectively. So, when a vehicle passes the red line, it is under the forbidden condition and that is how the violation detection process is going to be worked. But if a vehicle passes the green line, no step will be taken and the Video Capturing process will start again. After the Violation Detection process, an image of a violated vehicle is captured from the exact video frame. Selected Image data is processed by using many steps which are gray scaling, thresholding, canny edge detection and Contouring. By following the image processing steps, number plate of the vehicle will be found. If not found, the whole process is started from the step of the Capture Frame. When the plate region is found, that is extracted and prepared for the next step. After segmentation, predicted data is found as the recognized character.

\section{System Flow Chart for Violation Detection}

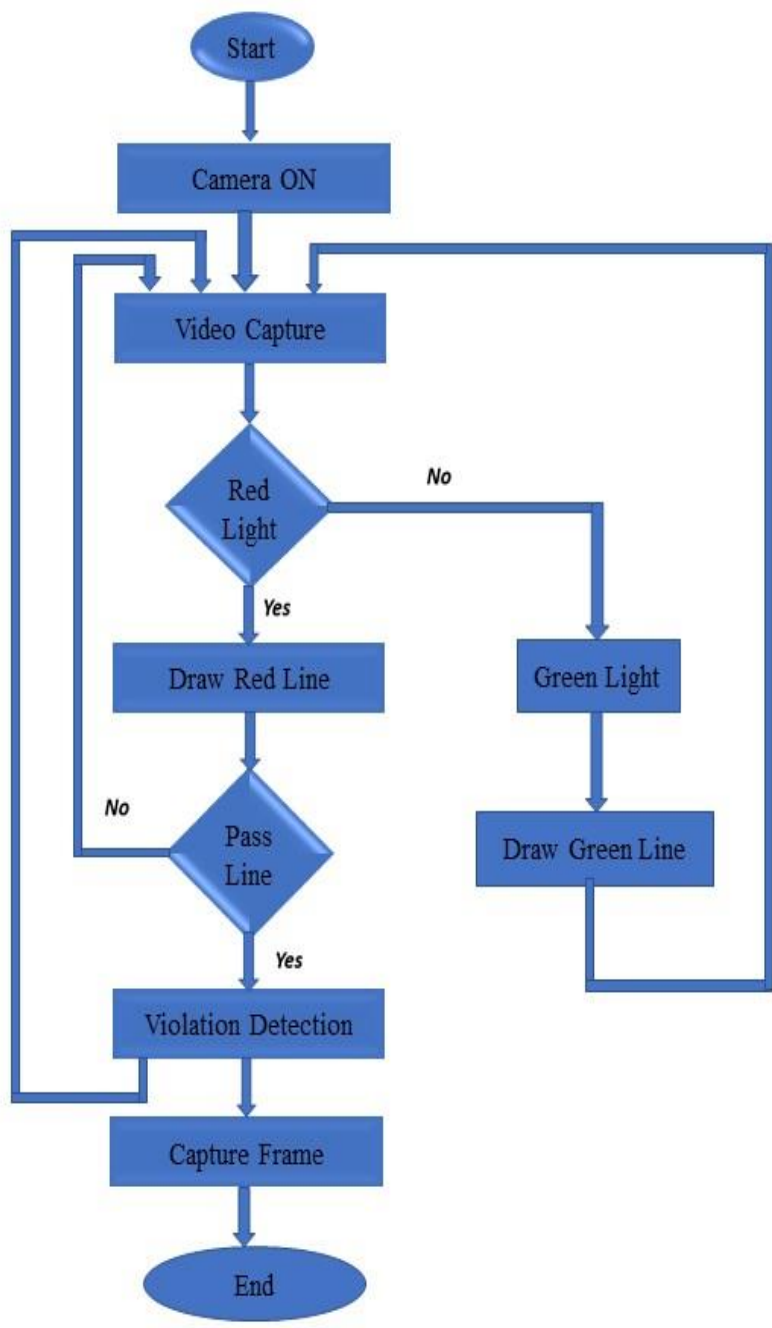

Fig. 3. System Flow Chart for Violation Detection
Automatic violation detection system flow chart is shown in figure 3 . This system includes capturing video, traffic signal, traffic signal violation detection and capture frame of the violated vehicle. Firstly, a video is captured on a road, while the red light/line of the traffic signal is ON. In the meantime, if any vehicle crosses the red light/line, it is detected as a violation of traffic rule. Finally, a frame is captured of this violated vehicle. The System was used two cameras, one was at a high angle to detect the violation and another one was at eye angle to capture the image. The eye angled camera was received command from the high angled camera to capture an image of a vehicle after the violation.

\section{System Flow Chart for ANPR}

In figure 4 , the flow chart of the ANPR system is shown. This system includes video data collection, frame capturing, image processing, number plate region finding, plate extraction and character recognition. The very important step is to find the plate region by the steps of image proposing, when, the character of number plate recognition is conformed to the data set of character.

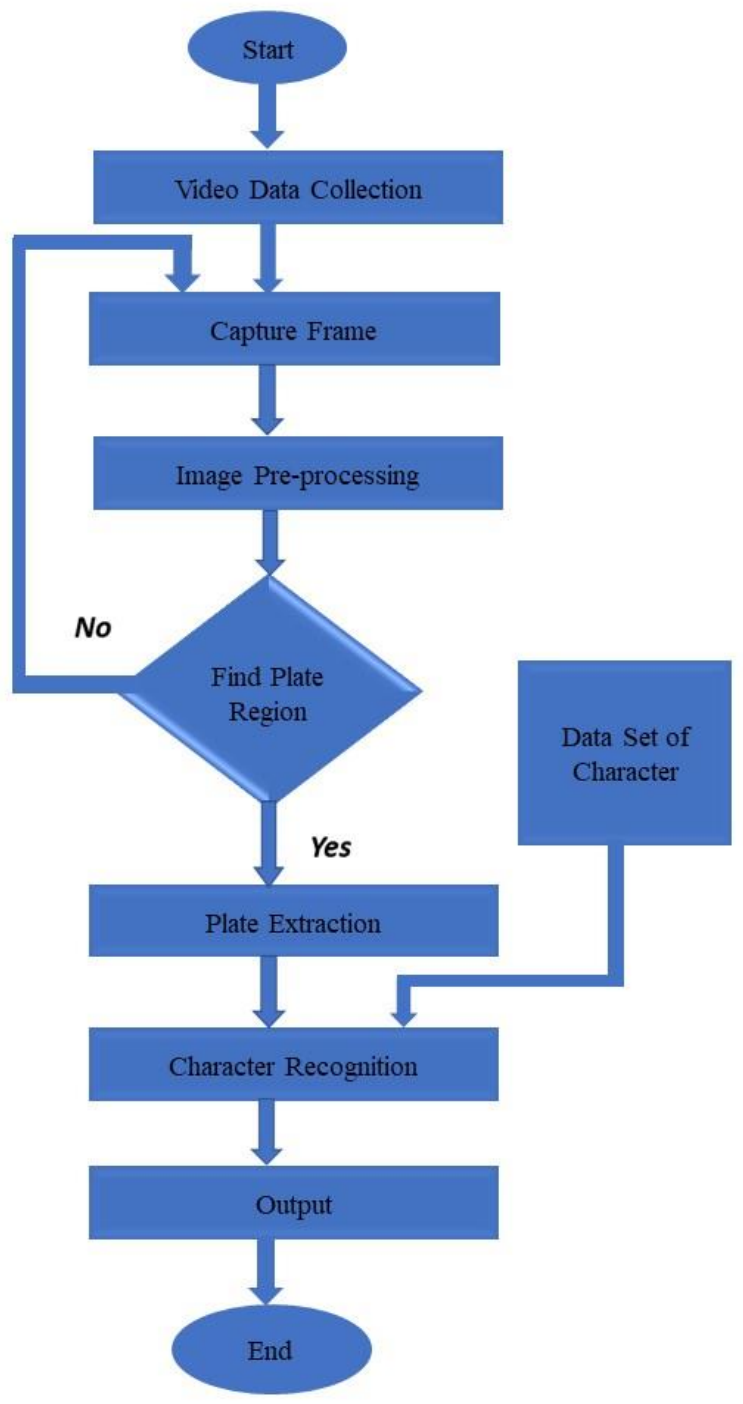

Fig. 4. System Flow Chart for Automatic Number Plate Recognition 
In the end, the final output results are showing. The code is designed enough smart to find the number plate from any position from wherever it is.

\section{Implementation Of The Project}

\section{A. Object detection}

The whole system was implemented using the Python programming language. YOLOv3, OpenCV and MATLAB were used for object detection, traffic rules violation detection

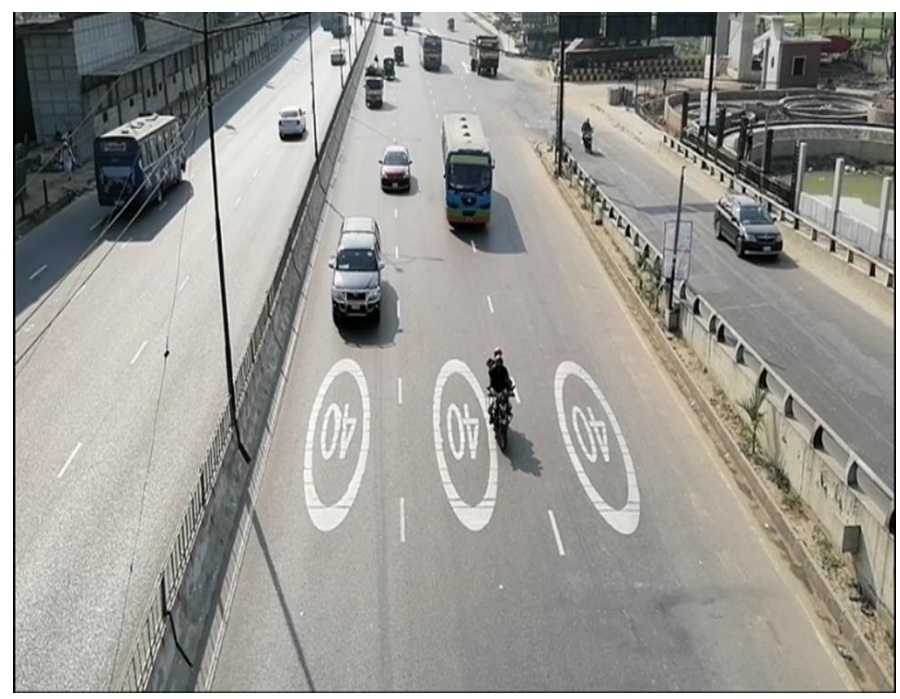

Fig. 5. Sample image for object detection (day)

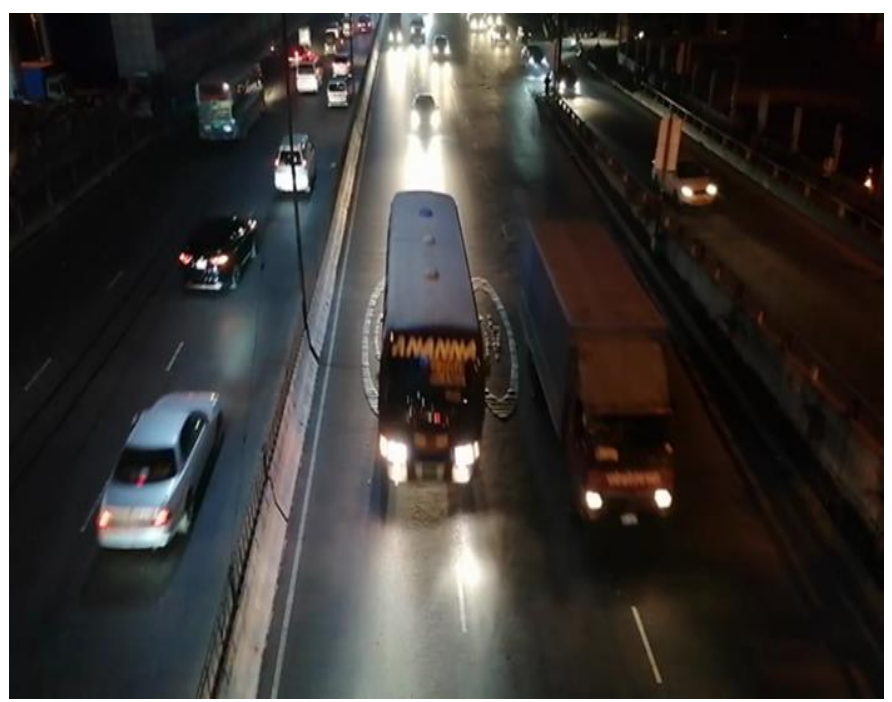

Fig. 6. Sample image for object detection (night)

and number plate recognition respectively. To recognize vehicles on the road object detection system was implemented using the YOLOv3 algorithm. For this purpose, the Linux operating system was chosen. YOLOv3 has its default trained data to detect various object including vehicles.

\section{B. Red light violation detection}

To detect red light violation video was captured by placing a camera right position of the road. After capturing video, a line was drawn by using the OpenCV library on the road indicating traffic signals where vehicles are supposed to be stopped when the red light of the traffic signal is ON.

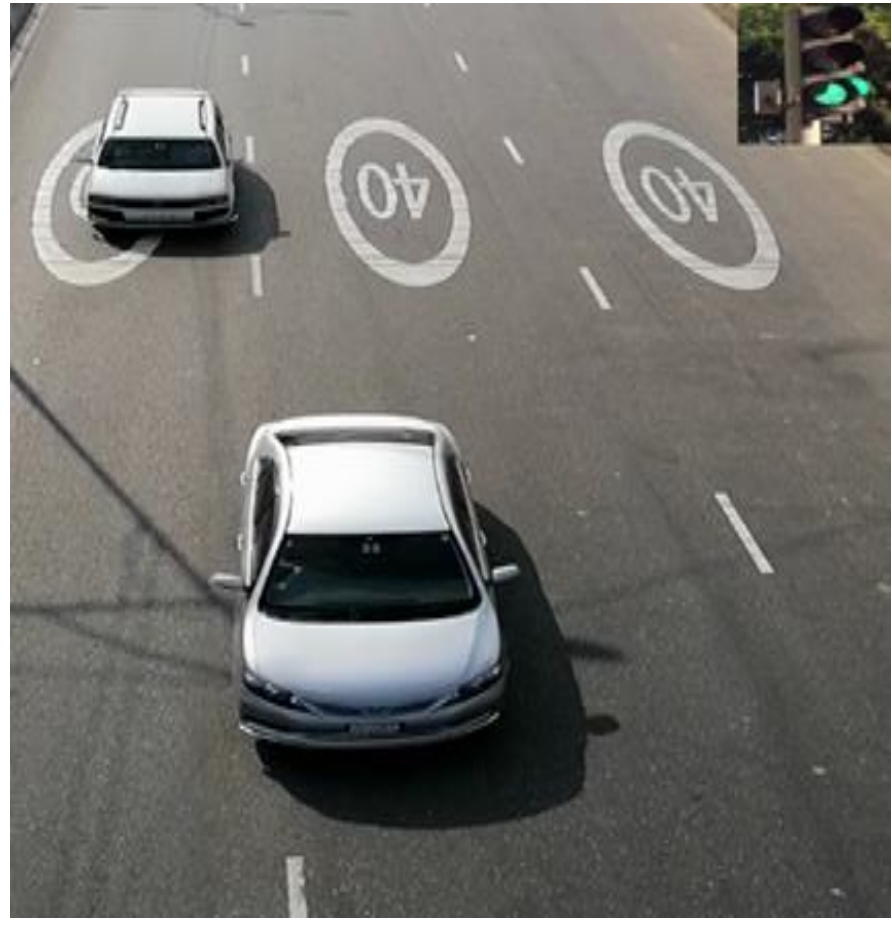

Fig. 7. Sample image for traffic Signal violation detection (day)

When a vehicle disobeyed the traffic signal bypassing the red line it was detected as the violated vehicle (car). To represent the green light of the traffic signal, the green line was drawn on the video. When a vehicle disobeyed the traffic signal bypassing the red line it was detected as the violated car. To represent the green light of the traffic signal, the green line was drawn on the video.

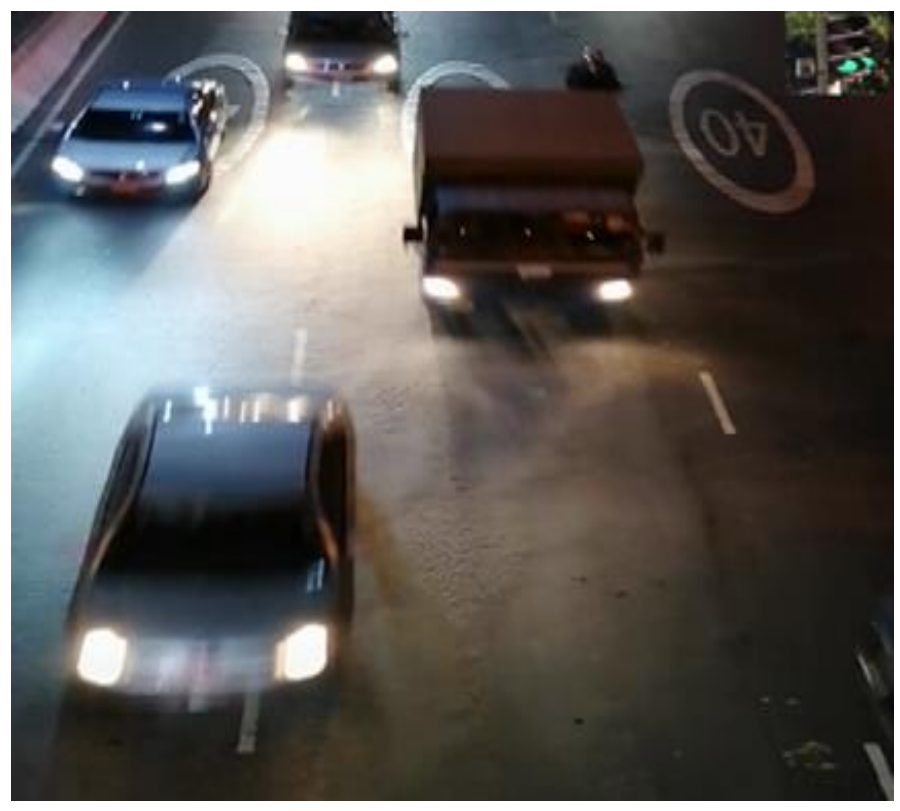

Fig. 8. Sample image for traffic Signal violation detection (Night)

\section{ANPR system}

The image was captured from the front of a vehicle of the higher elevation during traffic rule violation by a car and considering the camera was at a higher altitude and mounted on top of the traffic light. With the help of OpenCV certain 
processes were followed to detect the number plate. At first, the original image was converted into grayscale and then thresholding was done. By applying canny edge detection, the noise of the processed image was reduced by contouring twice. By following these algorithms, the region of interest of the number plate was found. After recognition of the number plate, characters of number plate were found using MATLAB. The system used OpenCV and MATLAB both. These functionalities were achieved by using "matlab.engine" in python platform to call MATLAB code.

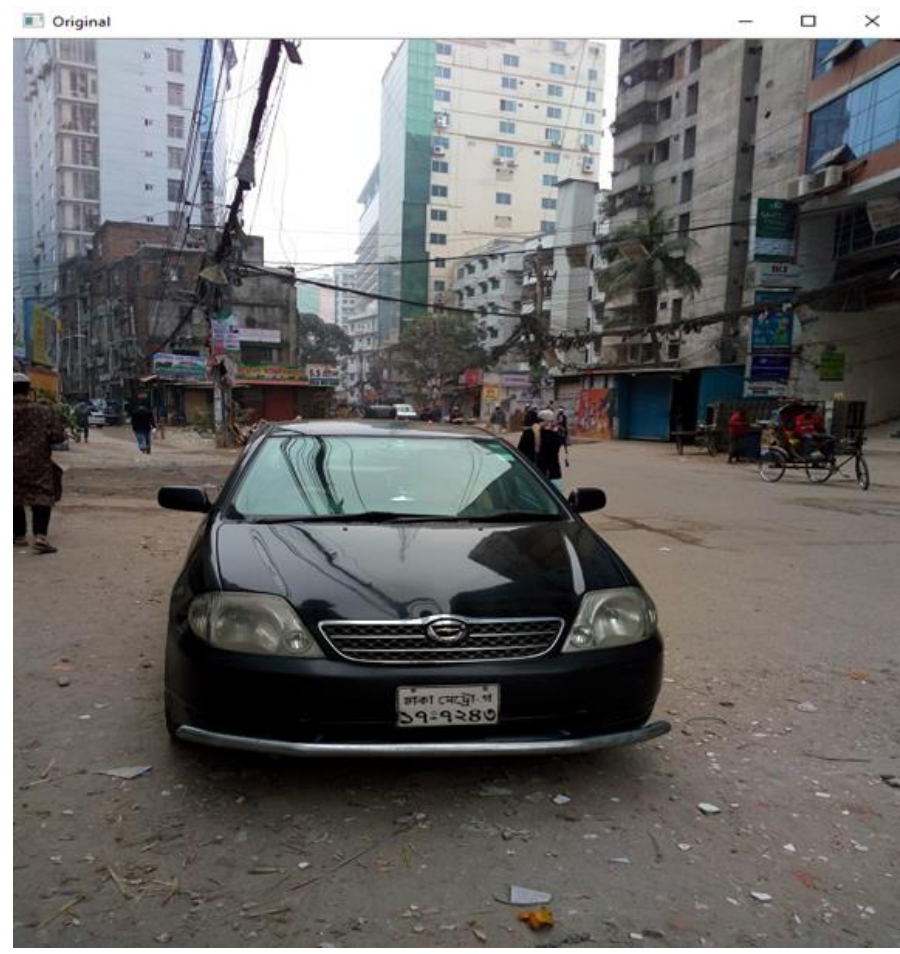

Fig. 9. Captured Image of a violated car (Day)

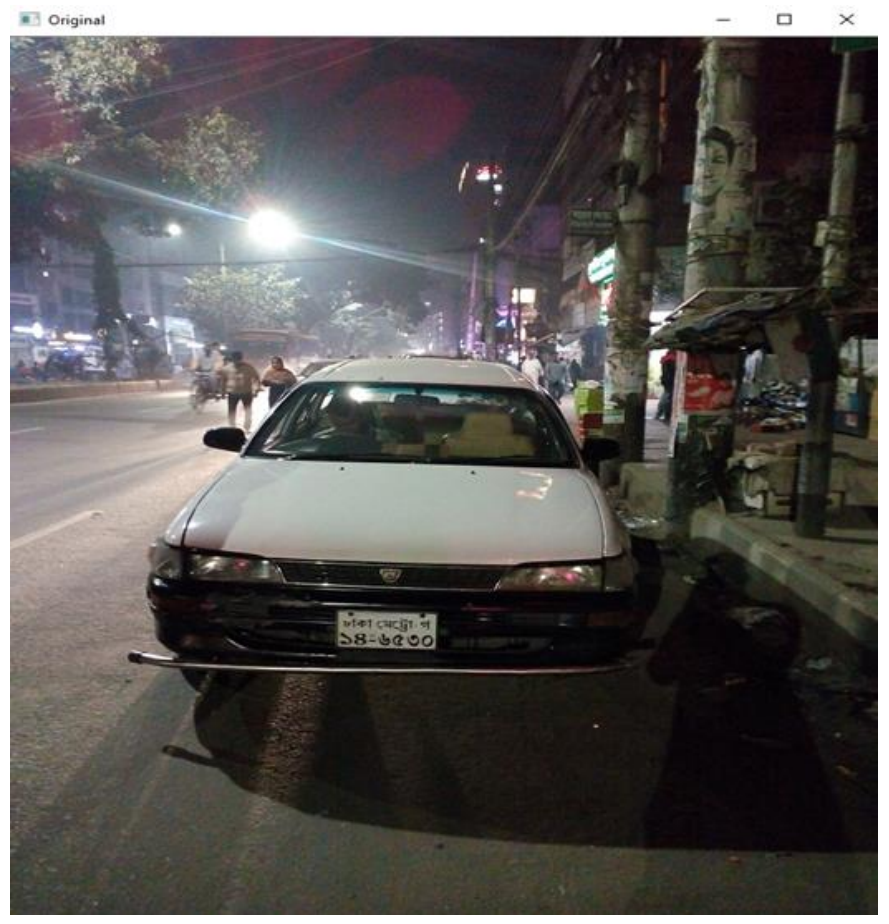

Fig. 10. Captured Image of a violated car (Night)

\section{OUTPUT DETECTION}

\section{A. Object detection}

For real-time situation, the camera price was very expensive, that's why pre-recorded video was used for object detection where frame rate was $30 \mathrm{fps}$ and the video duration was 11 seconds. YOLO v3 algorithm was applied in the Linux platform. Using the default data set of YOLOv3 various objects were detected including vehicles and humans on the road. The percentage of successful detection rate for cars is $80-99 \%$, for motorbike $66-89 \%$, for bus $94-100 \%$, bicycle $67-$ $89 \%$ and for person $60-88 \%$. The total processing time is 83 seconds. When the distance between the objects and the camera is decreased, the percentage rate will be increased.

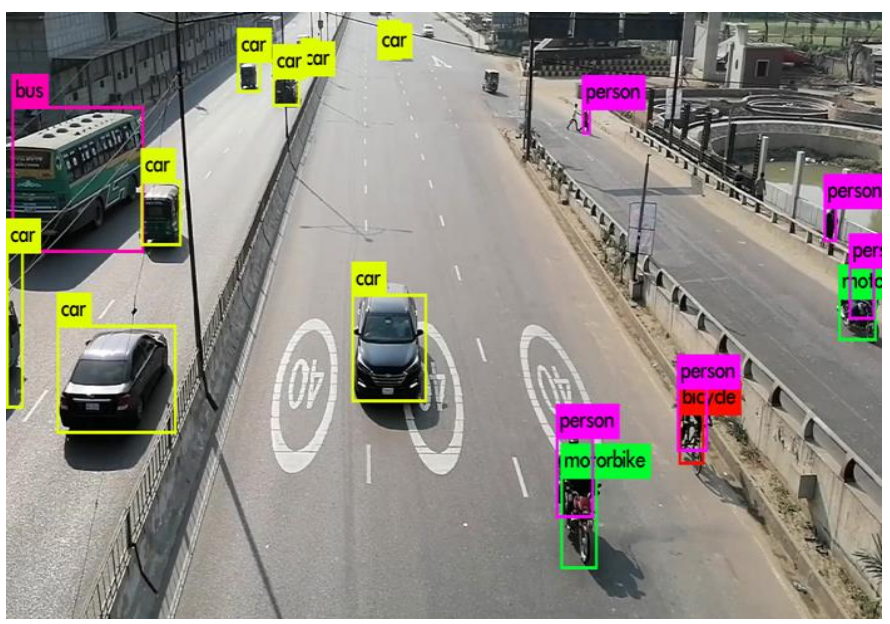

Fig. 11. Object detection using YOLOv3 (Day)

Three individual videos were taken at night time, to analyze the system's reliability at that time. The achieved result was outstanding. Though the system took a long time to detect which was average 69.92 seconds, the accuracy was very good. The range of accuracy for the bus is $82-92 \%$, for the car is $81.20-93.83 \%$, for truck the range of accuracy is $80-87 \%$.

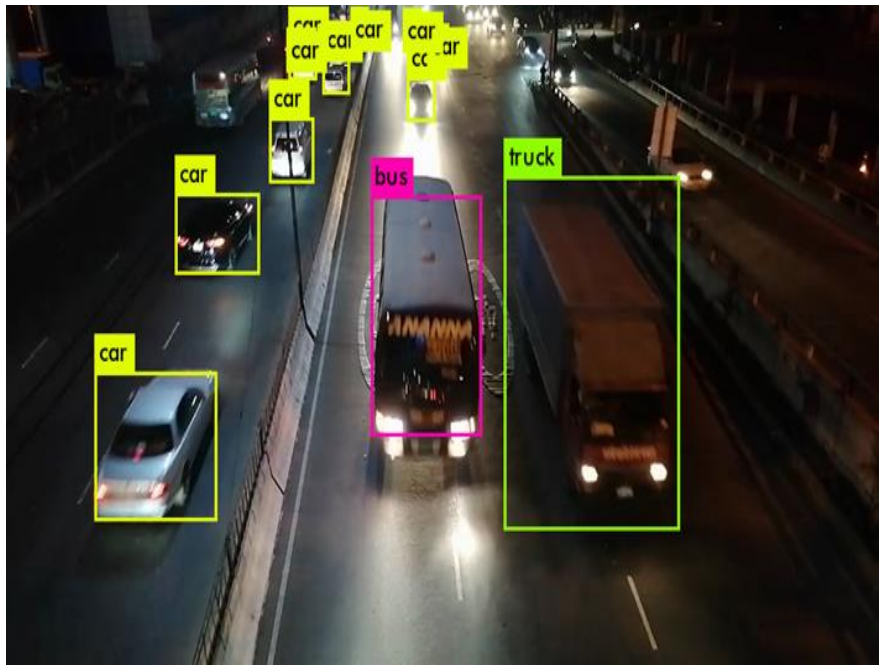

Fig. 12. Object detection using YOLOv3 (Night)

\section{B. Red light violation}

OpenCV libraries were used for violation detection purposes. A line was drawn using OpenCV when a car passed the line it was detected as the violated car. The video duration was 58 
seconds and the frame rate was $30 \mathrm{fps}$. Because of the price, higher resolution camera was not used for which frame rate can be affected. The detection time is 2.84 seconds where accuracy is over $90 \%$. On the other hand, accuracy at night time is almost $85 \%$, due to low light intensity.

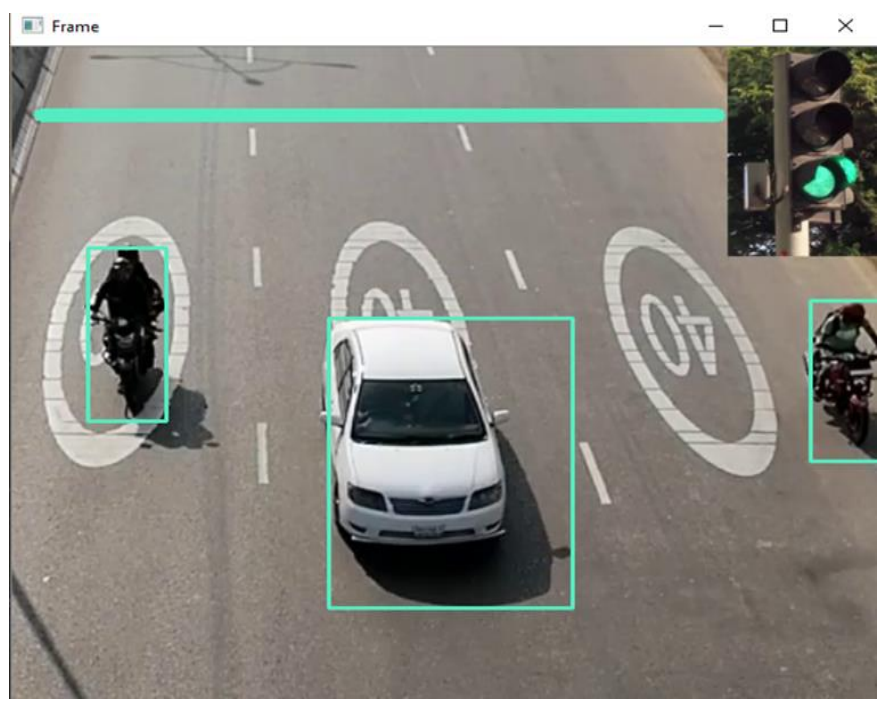

Fig. 13. When the green signal was drawn (Day)

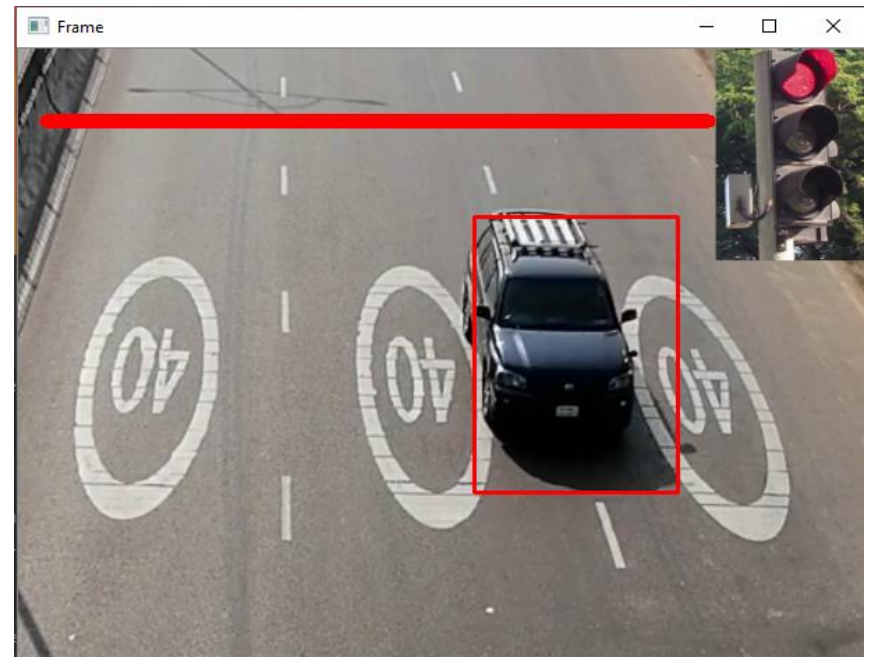

Fig. 14. Red light violation (Day)

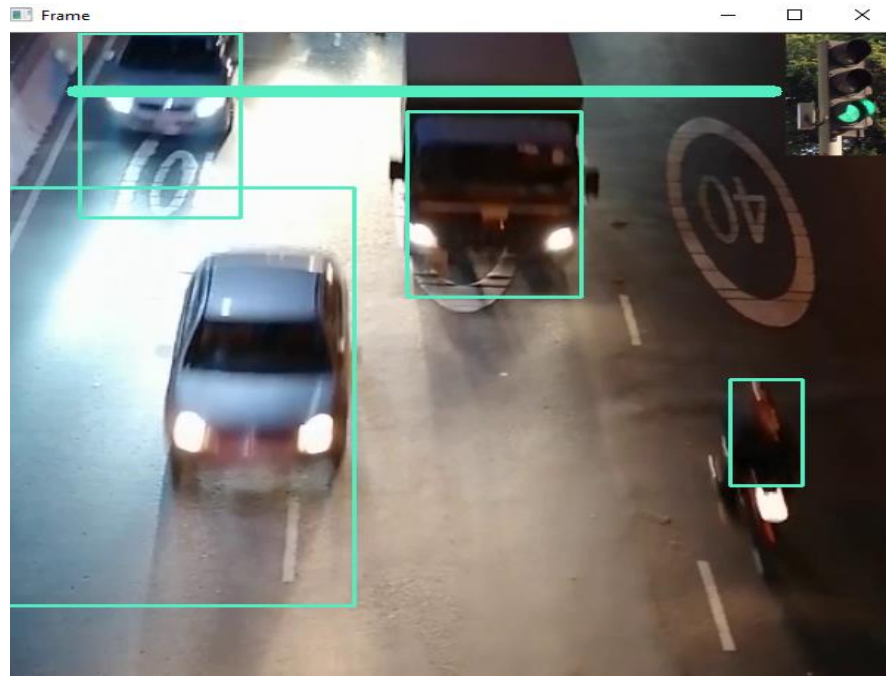

Fig. 15. When the green signal was drawn (Night)

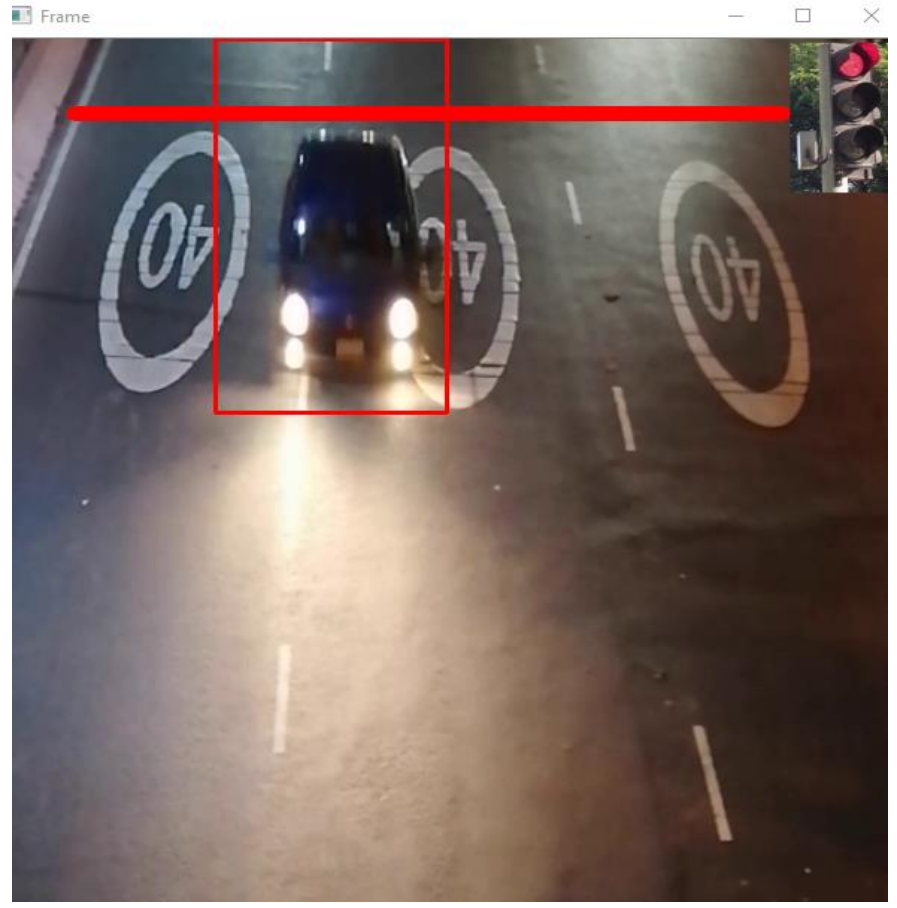

Fig. 16. Red light violation (Night)

\section{ANPR}

After detecting a violation, an image was captured for number plate recognition which dimension was $640 \times 480$. The total processing time was 3.85 seconds. The percentage of successful recognition is $98 \%$. The best result was found when the vehicle was 3.5 meters away from the camera horizontally. The camera was set 1.7 meters above from the ground.

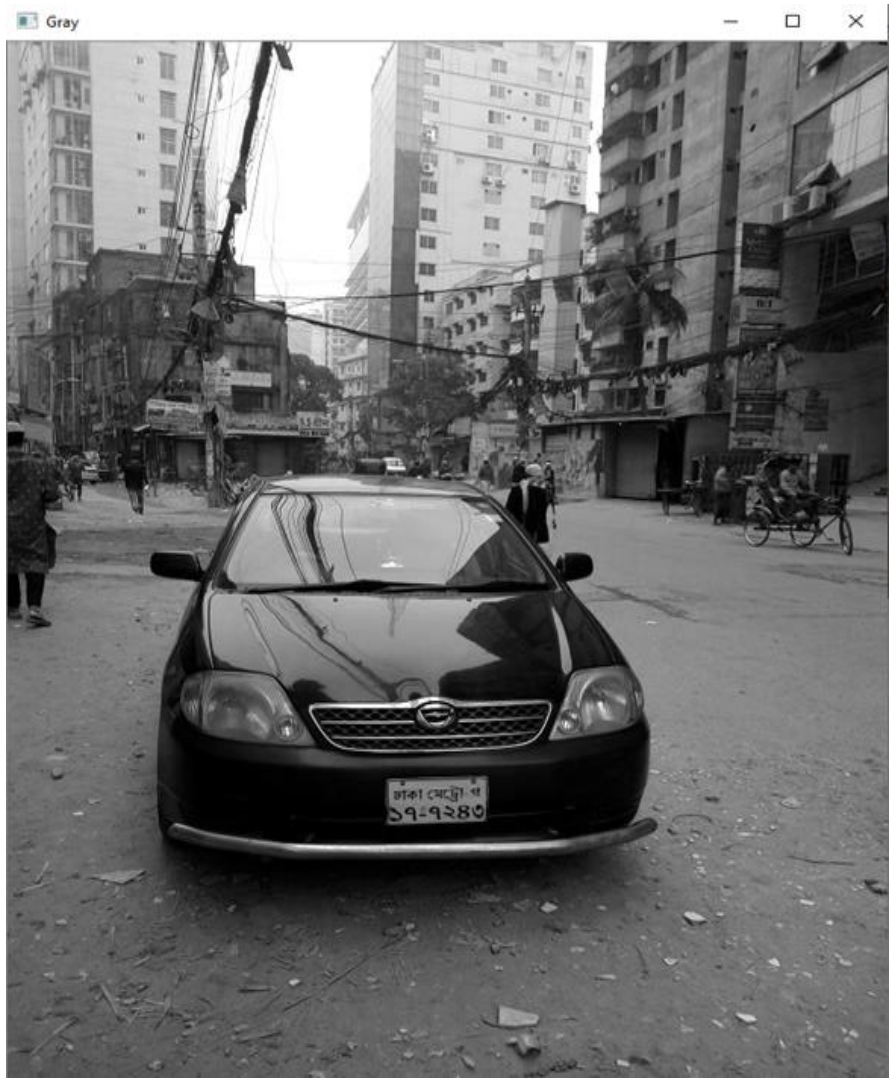

Fig. 17. Gray scaling of the captured image (Day) 

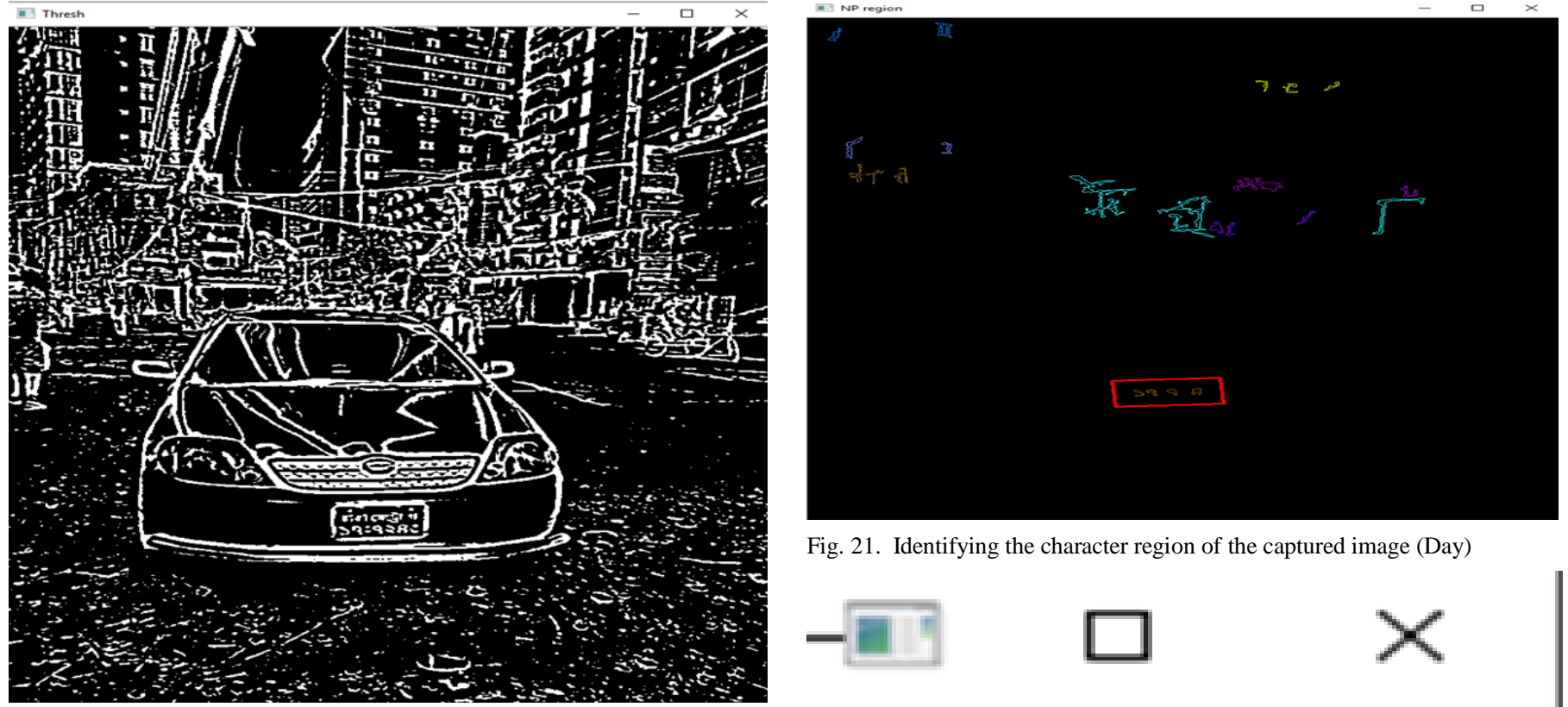

Fig. 21. Identifying the character region of the captured image (Day)

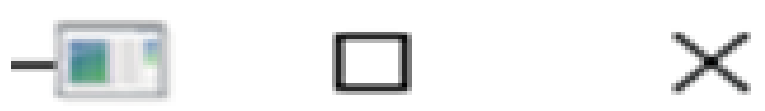

Fig. 18. Thresholding image of the captured image (Day)

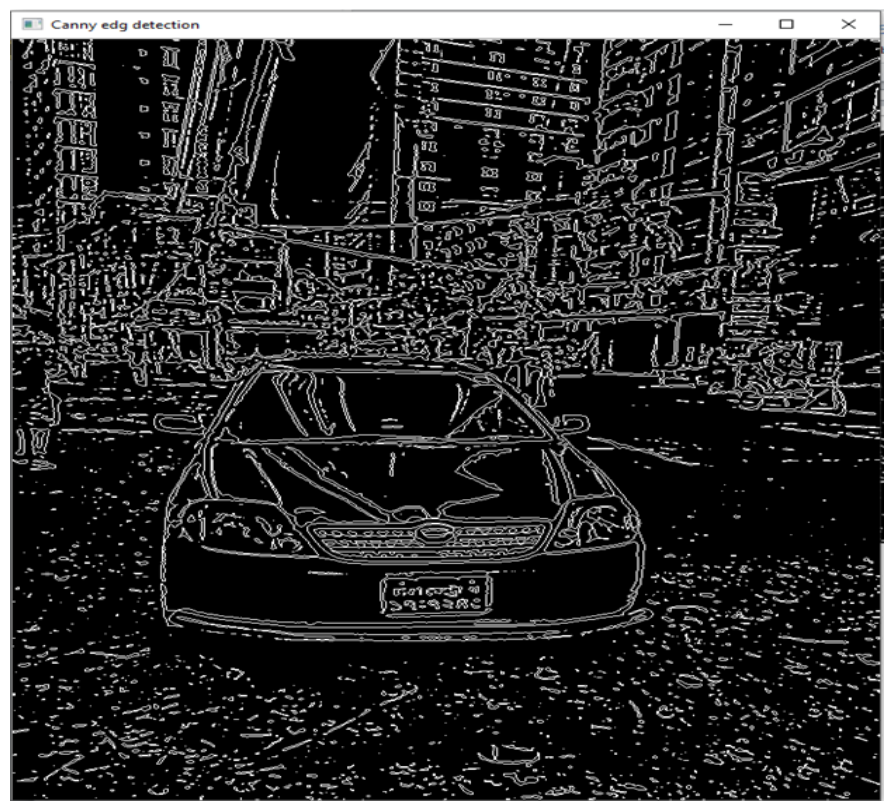

Fig. 19. Canny edge detection of the captured image (Day)

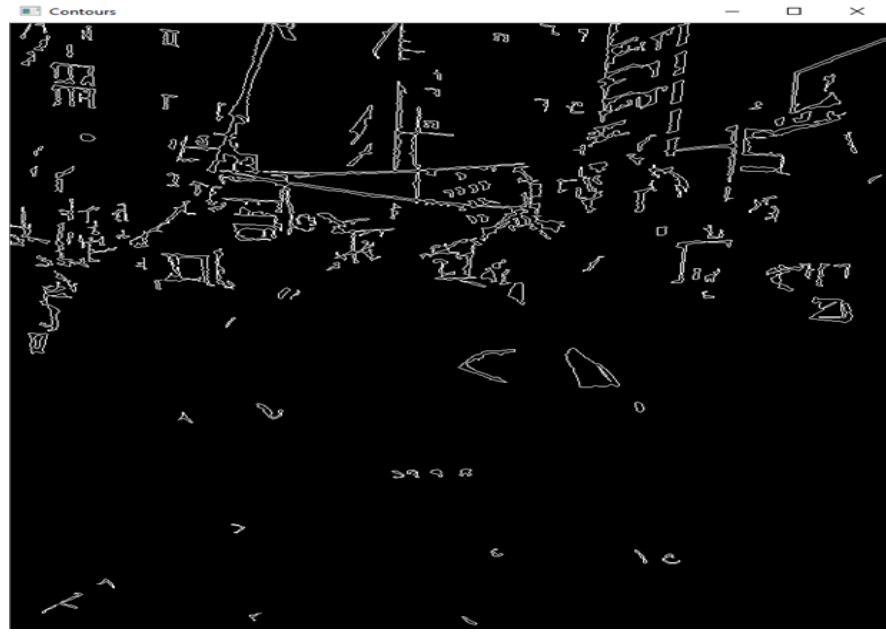

Fig. 20. Contouring to reduce the noise of captured image (Day)

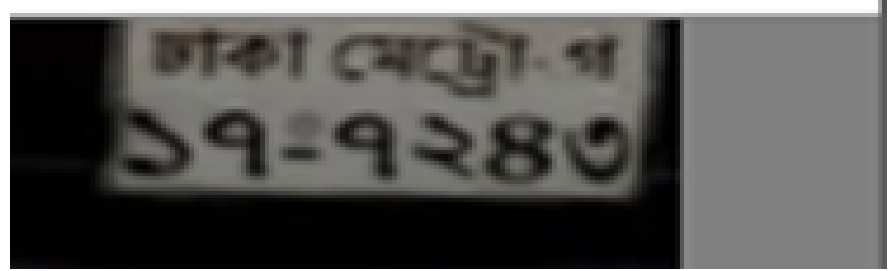

Fig. 22. Possible number plate extraction of the captured image (Day)

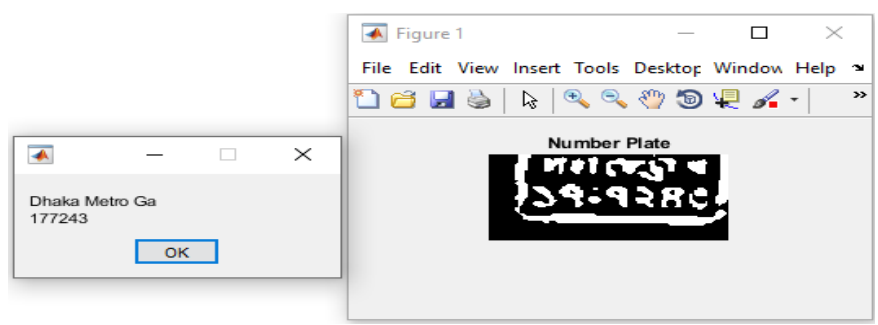

Fig. 23. Character recognition (Day)

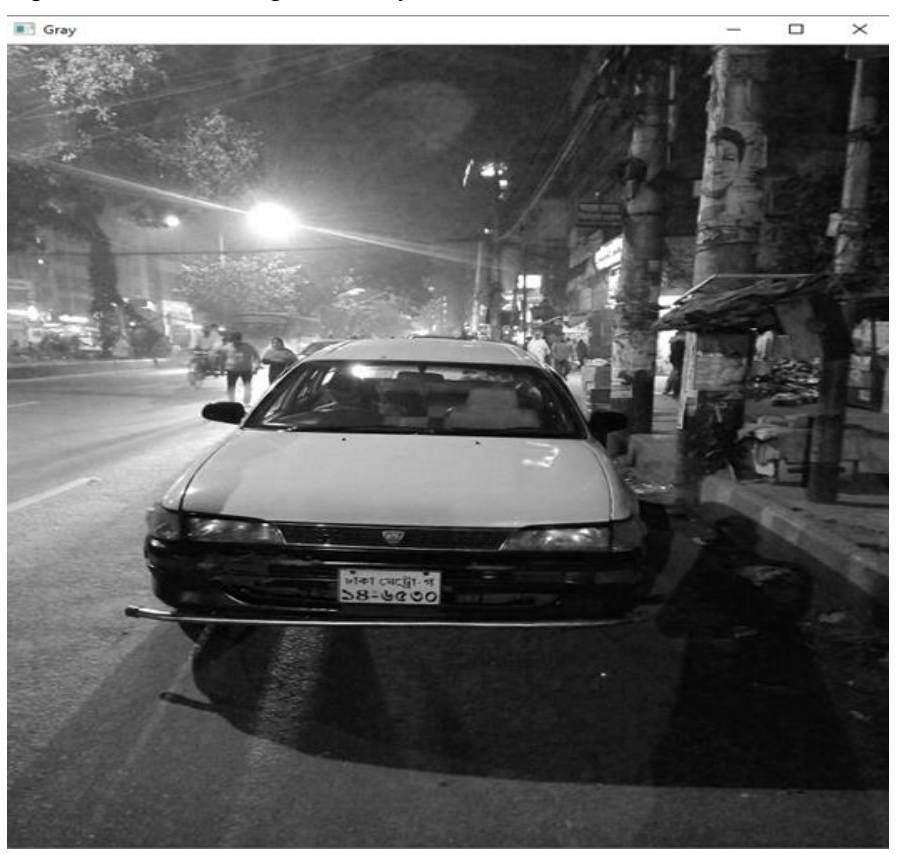

Fig. 24. Gray scaling of the captured image (Night) 


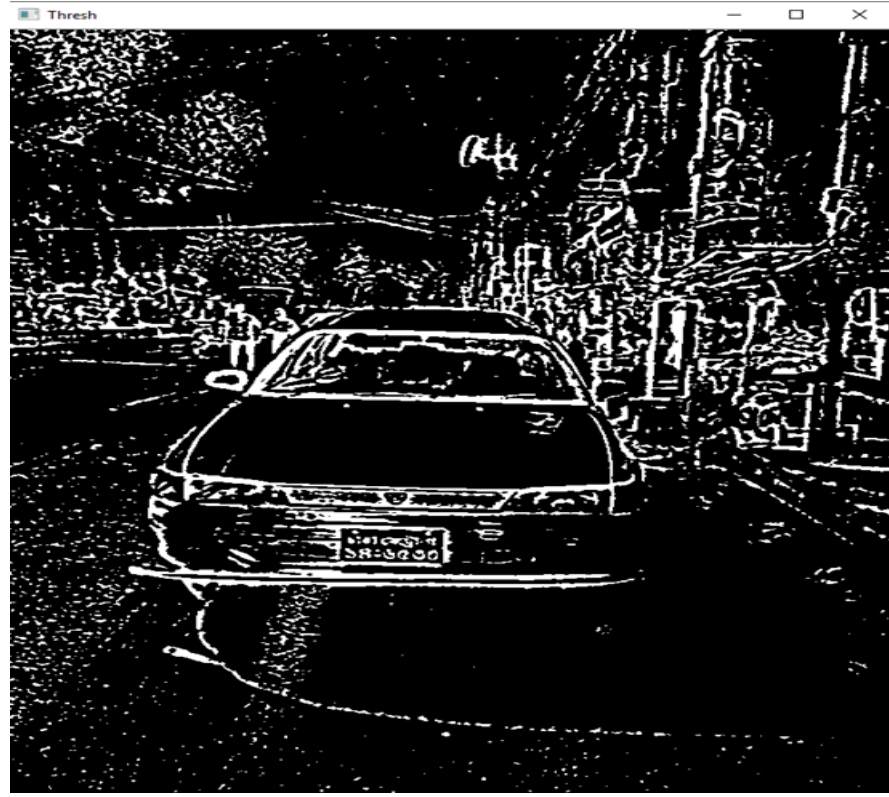

Fig. 25. Thresholding image of the captured image (Night)

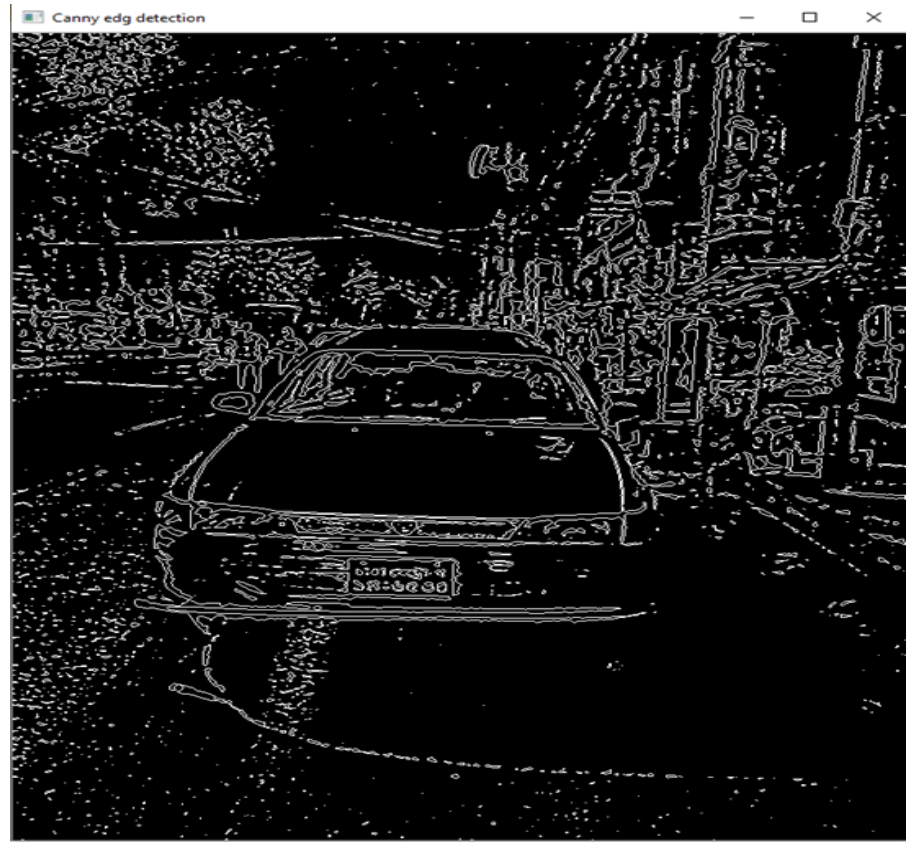

Fig. 26. Canny edge detection of the captured image (Night)

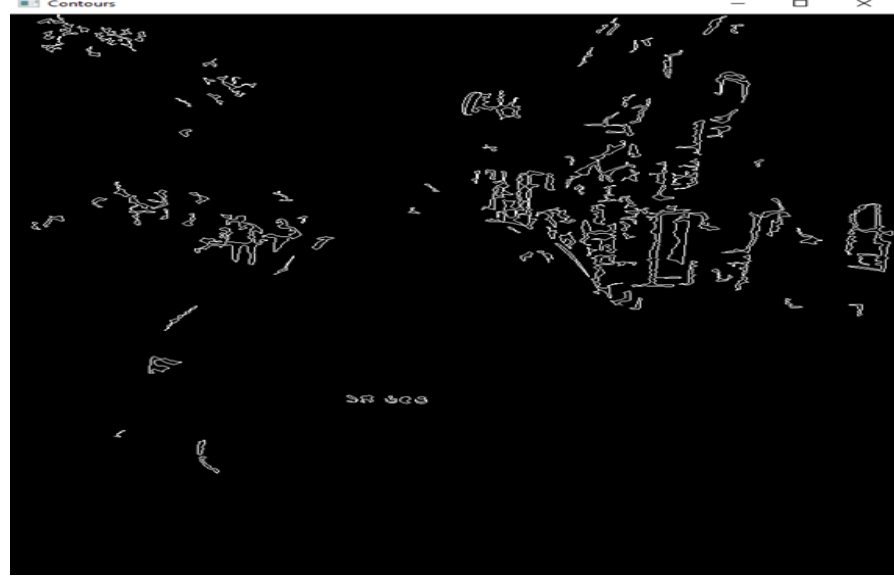

Fig. 27. Contouring to reduce the noise of captured image (Night)

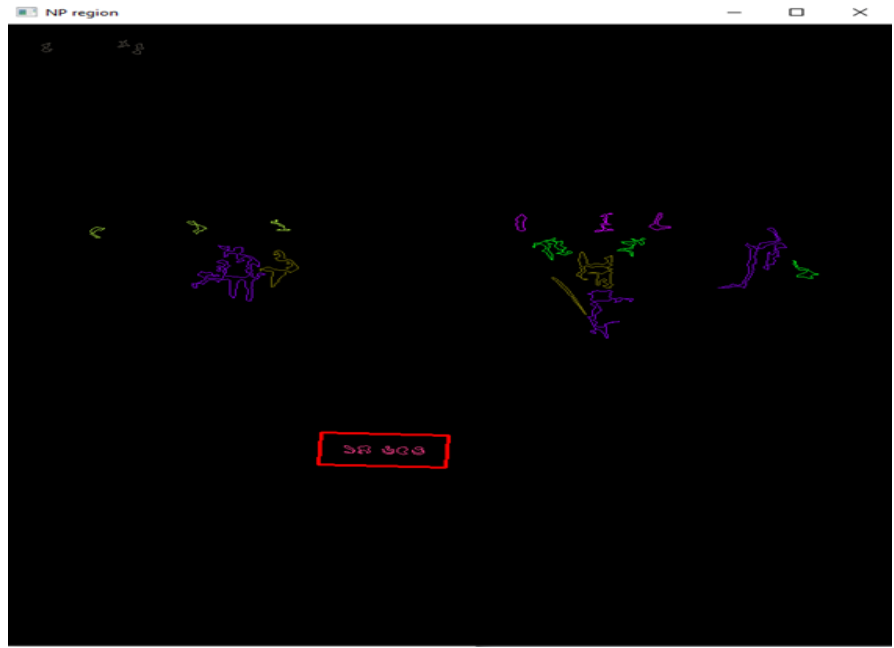

Fig. 28. Identifying the character region of the captured image (Night)
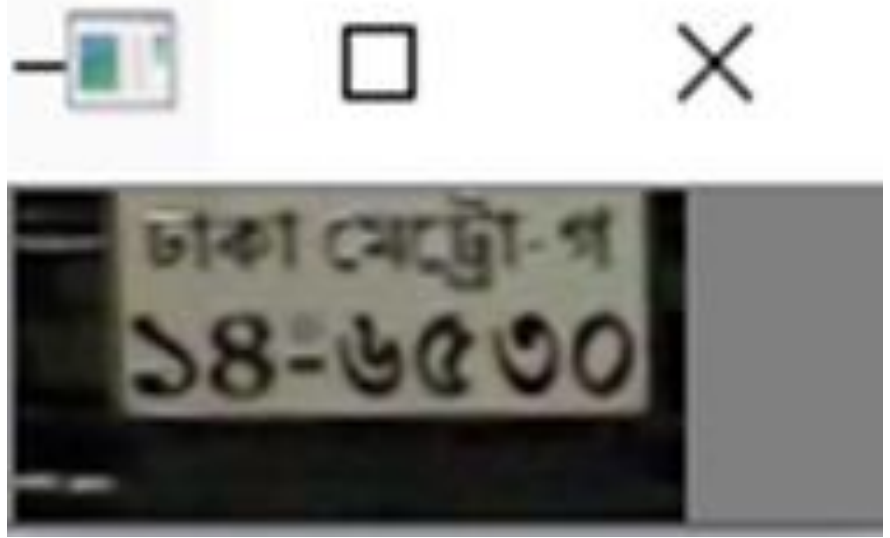

Fig. 29. Possible number plate extraction of the captured image (Night)

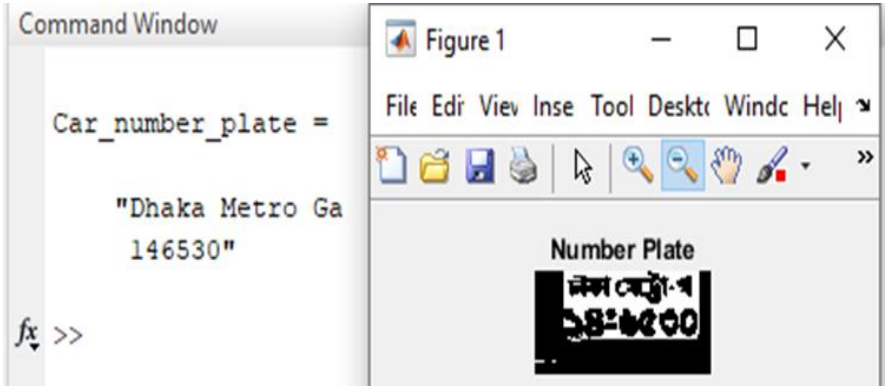

Fig. 30. Character recognition from number plate (Night)

\section{Result Analysis}

After training those video footages the ratio of the confidence value may vary from time to time. But the highest confidence value is assured by training \& giving proper acquisition of time. Sometimes information of the identified may overlap with others. But proper information that is provided in the database is retrieved at the end. From the captured video by using the YOLO algorithm, 5 samples were taken based on distance from the camera to objects on the road. A total of 5 samples were analyzed and it was found that sample 4 provided the best result. Based on the highest accuracy, sample 4 was best because the objects were near to the camera and daylight was sufficient. The highest accuracy of detection for bus $99 \%$, for a person $81 \%$, for car $94 \%$, for 
motorbike $69 \%$, and bicycle $61 \%$. If the large data set of motorbike and bicycle could be used, the accuracy was up to the mark.

Table I

Percentage of Object Detection data (Day)

\begin{tabular}{|c|c|c|c|c|c|}
\hline Sample & Bus & Person & Car & Motorbike & Bicycle \\
\hline Sample 1 & $91.66 \%$ & $67.25 \%$ & $94 \%$ & $59 \%$ & $59 \%$ \\
\hline Sample 2 & $91.66 \%$ & $81 \%$ & $83.36 \%$ & $69 \%$ & $59 \%$ \\
\hline Sample 3 & $99 \%$ & $64.33 \%$ & $87.57 \%$ & $69 \%$ & $58 \%$ \\
\hline Sample 4 & $99 \%$ & $72.50 \%$ & $80.40 \%$ & $69 \%$ & $61 \%$ \\
\hline Sample 5 & $93 \%$ & $60 \%$ & $75 \%$ & $69 \%$ & $61 \%$ \\
\hline
\end{tabular}

\section{Accuracy of Object Detection (\%) Vs Object Name}

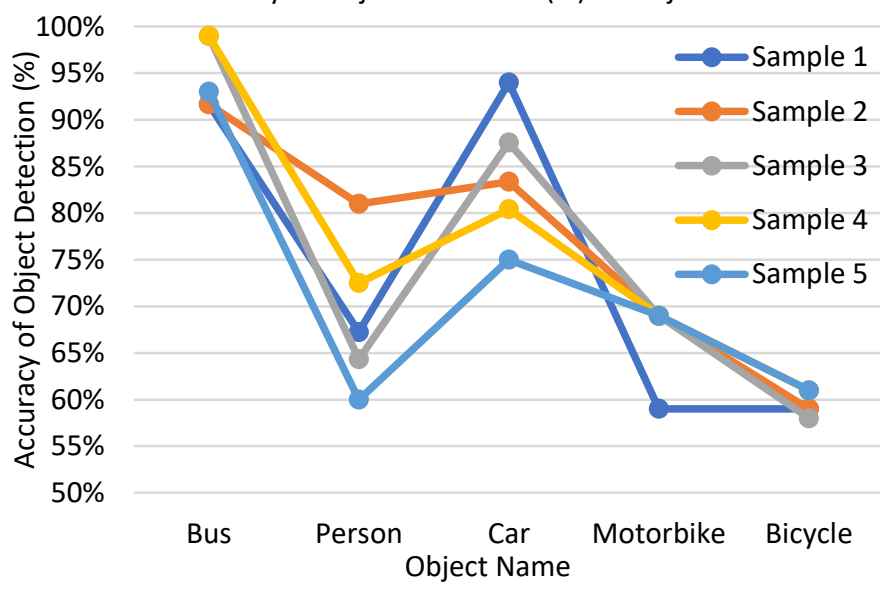

Fig. 31. Accuracy of Object Detection (\%) Vs Object Name (Day)

Table II

Best Percentage of Object Detection data (Day)

\begin{tabular}{|c|c|c|c|c|c|}
\hline Sample & Bus & Person & Car & Motorbike & Bicycle \\
\hline Sample 4 & $99 \%$ & $72.50 \%$ & $80.40 \%$ & $69 \%$ & $61 \%$ \\
\hline
\end{tabular}

Accuracy of Object Detection (\%) Vs Object Name

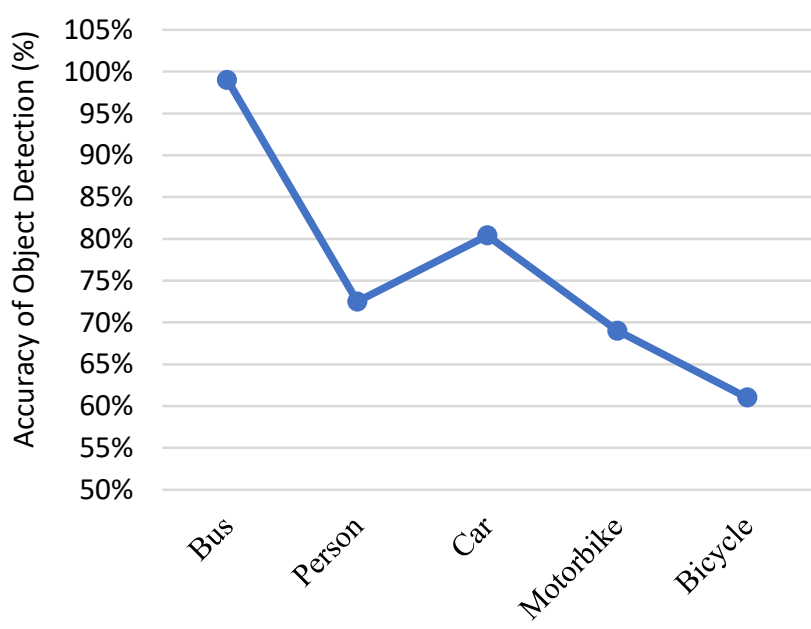

Object Name

Fig. 32. Best Object Detection Data (Day)
From the captured video by using the YOLO algorithm, 3 samples were taken based at night on distance from the camera to objects on the road. A total of 3 samples were analyzed and it was found that sample 1 provided the best result.

Table III

Percentage of Object Detection data (Night)

\begin{tabular}{|c|c|c|c|}
\hline Sample & Bus & Truck & Car \\
\hline Sample 1 & $98 \%$ & $80 \%$ & $93.83 \%$ \\
\hline Sample 2 & $82 \%$ & $81.2 \%$ & $81.20 \%$ \\
\hline Sample 3 & $82 \%$ & $80 \%$ & $83.93 \%$ \\
\hline
\end{tabular}

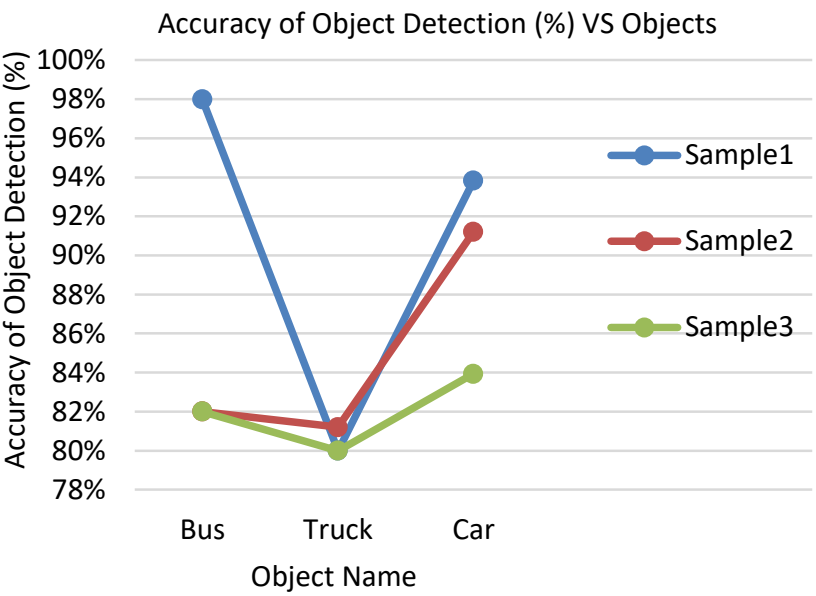

Fig. 33. Accuracy of object detection over different vehicles (Night)

Based on the highest accuracy, sample 1 was the best because the objects were near to the camera and for enough intensity of street light at night. The highest accuracy of detection for bus $98 \%$, for truck $80 \%$ and car $93.83 \%$.

Table IV

Best Percentage of Object Detection Data (Night)

\begin{tabular}{|l|l|l|l|}
\hline Sample & Bus & Truck & Car \\
\hline Sample 1 & $98 \%$ & $80 \%$ & $93.83 \%$ \\
\hline
\end{tabular}

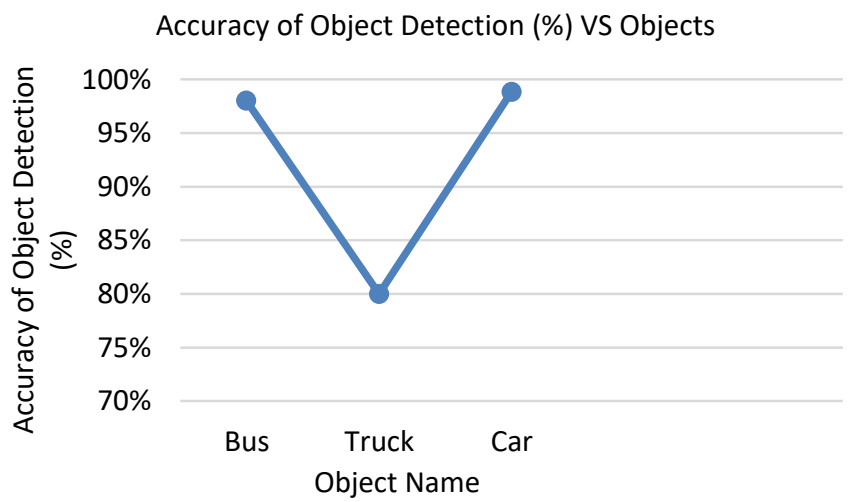

Fig.34. Accuracy of best object detection over different vehicles (Night)

Few figures and graphs are helping to analysis the outcome of the research. These data are gathered in two different times 
like, day and night. Among these, the data of day is clearer and more perfect with respect to night due to light intensity. However, the situation can be solved either by setting up enough street light with high intensity or using a highresolution night vision camera and this thing will be worked at fogy or a rainy day too.

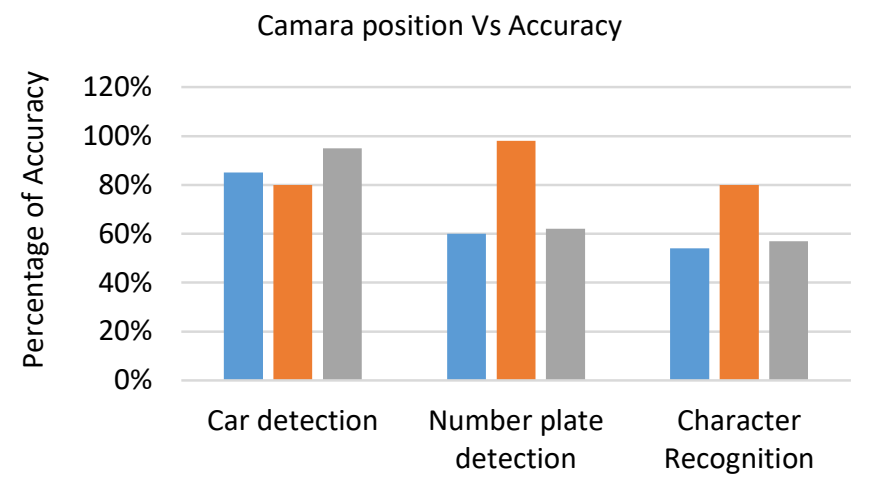

- Sample 1 Sample 2 Sample 3

Fig. 35. Camera Position vs Accuracy (Day)

Figure 35, shows the accuracy of operations based on samples that were taken from a different angle of camera position. Here sample1, sample2 and sample3 were sequentially represented low angle, eye angle and high angle. Accuracy of Car detection, Number plate detection and character recognition of eye angle was $95 \%, 98 \%$ and $80 \%$ respectively in the day time. Result of Road object detection and violation detection was taken from the high angle and number plate recognition was done from the eye angle. From the data between day and night, data of day were shown the good accuracy because of sufficient light intensity.

Table VI

Result analysis

\begin{tabular}{|c|c|c|c|l|}
\hline Event & $\begin{array}{c}\text { Detection } \\
\text { Accuracy } \\
\text { (Day) }\end{array}$ & $\begin{array}{c}\text { Detection } \\
\text { Accuracy } \\
\text { (Night) }\end{array}$ & $\begin{array}{c}\text { Total Process } \\
\text { Time Required } \\
(\mathrm{sec})\end{array}$ & $\begin{array}{c}\text { Camera } \\
\text { Position }\end{array}$ \\
\hline $\begin{array}{c}\text { Road Object } \\
\text { Detection }\end{array}$ & $99 \%$ & $90.61 \%$ & 74.51 & High Angle \\
\hline $\begin{array}{c}\text { Violated Car } \\
\text { Detection }\end{array}$ & $95 \%$ & $85 \%$ & 2.84 & High Angle \\
\hline $\begin{array}{c}\text { Number Plate } \\
\text { Detection }\end{array}$ & $98 \%$ & $80 \%$ & 3.85 & Eye Angle \\
\hline $\begin{array}{c}\text { Character } \\
\text { Recognition }\end{array}$ & $80 \%$ & $75 \%$ & 3.50 & - \\
\hline
\end{tabular}

Available technology what is existed actually based on machine learning and little more sensors. Now, technology is advancing and turning on fully machine learning and IoT based and this research was actually focused on this advanced technology and no sensor was used only processing device, cameras, and python programming. Another good thing in this research was image processing time where red light violation and automatic number plate recognition (ANPR) were taken $2.84 \mathrm{sec}$ and $3.85 \mathrm{sec}$ respectively, that was enough processing speed to complete this process and if consider the accuracy which was also quite well around 80 to 90 per cent. The image processing speed and accuracy can be improved, what if a good processing unit is used and large data set can be generated respectively.

\section{Conclusion}

In this research, Automatic traffic rules violation detection and number plate recognition system is presented. Image processing techniques are used to develop this system. YOLOv3 was used here to detect the object on the road. Using OpenCV the traffic rules violation was detected. MATLAB was used to recognize the characters of the number plate. Where object detection, violation detection and number plate reorganization were done sequentially for day 99\%, 95\%, $80 \%$ and also sequentially for night $90.61 \%, 85 \%, 75 \%$. The results were evaluated on light intensity and camera position. The accuracy of the system was more accurate in day time due to higher light intensity than night time. The system is highly sensitive to the position of the camera. The smaller the distance between the camera and the vehicles the higher the detection accuracy. At an optimum angle, the accuracy was highest. This visibility problem like different weather condition such as rain, storm, and bad visibility due to dust or dusty number plate can be solved through a higher resolution camera and thousands of data of number plate.

\section{REFERENCES}

[1] T. Stone, "A complete history of the traffic signal", Dec., 2017. [Online].Available:https://www.traffictechnologytoday.com/feat ures/a-complete-history-of-the-traffic-signal.html [Accessed Aug. 18,2019].

[2] M. Fabry, "This Is Why Cars Have License Plates", Apr. 25, 2016. [Online]. Available: https://time.com/4301055/numberplate-history/ [Accessed Aug. 18,2019]

[3] Joarder, M.M.A., Mahmud, K., Tasnuva, A., Kawser, M. and Ahamed, B., 2012. Bangla automatic number plate recognition system using artificial neural network, pp.1.

[4] Sharma, Rinky. "Automatic license plate based smart vehicle validation \& security by gate control \& email send." International Journal of Computer Science and Information Technologies 6.2 (2015): 952-957.

[5] D. Madhu Babu, K. Manvitha, M. S. Narendra, A. Swathi, K. Praveen Varma, "Vehicle Tracking Using Number Plate Recognition System." International Journal of Computer Science and Information Technologies 6.2 (2015): 1473-1476.

[6] R. Anderson, "Dubai's RTA Confirms Dhs540m project to expand smart traffic system", Nov., 2018. [Online]. Available:https://gulfbusiness.com/dubais-rta-confirmsdhs590m-project-expand-smart-traffic-system/ [Accessed Sept. 10,2020].

[7] J. Canny, "A Computational Approach to Edge Detection", IEEE Transaction on Pattern Analysis and Machine Intelligence, Vol. 8, No. 6, 1986.

[8] Garg, N. and Garg, N., 2013. Binarization techniques used for grey scale images. International Journal of Computer Applications, 71(1), pp.8-11.

[9] Anbarjafari, G., 2014. Introduction to image processing. Digital Image Processing. University of Tartu, Tartu, pp.2-4.

[10] M. Bertozzi, A. Broggi, A. Fascioli, and S. Nichele, "Stereo Vision-based Vehicle Detection", in proceedings of IEEE Intelligent Vehicles Symposium, pp. 39-44, Dearbon, MI, USA, 2000 . 
[11] Sang, J., Wu, Z., Guo, P., Hu, H., Xiang, H., Zhang, Q., \& Cai, B. (2018). An improved YOLOv3 for vehicle detection. Sensors, 18(12), 4272.

[12] Thiel, G. (2000). Automatic CCTV surveillance-towards the VIRTUAL GUARD. IEEE Aerospace and Electronic Systems Magazine, 15(7), 3-9.

[13] Yi, Z., Yongliang, S. and Jun, Z., 2019. An improved tinyyolov3 pedestrian detection algorithm. Optik, 183, pp.17-23.

[14] Hasan, M., 2011. Real time detection and recognition of vehicle license plate in Bangla, pp.2-3.

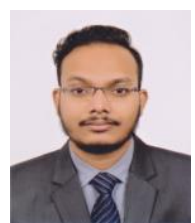

Raian Shahrear received his B.Sc. degree in Electrical and Electronic Engineering from American International University-Bangladesh in. His research interest includes Machine Learning, Artificial Intelligence (AI), and Internet of Things (IoT). Mr. Raian is a student member of the Institute of Electrical and Electronics Engineers (IEEE).

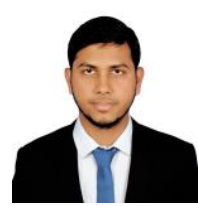

Md. Anisur Rahman received B.Sc. in Electrical and Electronic Engineering degree from American International University-Bangladesh (AIUB). His research interests are Artificial Intelligence (AI), Machine Learning, and Renewable Energy. Mr. Anisur is a student member of the Institute of Electrical and Electronics Engineers (IEEE).

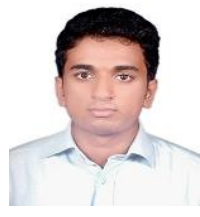

Atif Islam received his B.Sc. in Electrical and Electronic Engineering from American International University-Bangladesh (AIUB). $\mathrm{He}$ is a student member of Institute of Electrical and Electronic Engineers (IEEE). His research interest includes Signal Processing, Robotics, AI and Machine

Learning.

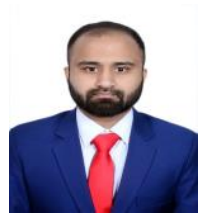

Chamak Dey received his B.Sc. in Electrical and Electronic Engineering from American International University-Bangladesh (AIUB). $\mathrm{He}$ is a student member of Institute of Electrical and Electronic Engineers (IEEE). His research interest includes chip designing and Machine learning.

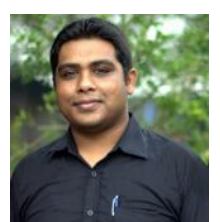

Md. Saniat Rahman Zishan received B.Sc. in Electrical and Electronic Engineering and Master of Engineering in Telecommunications degree from American International University-Bangladesh (AIUB). On September 2009, he started his teaching career as a lecturer in AIUB. At present he is serving as an Associate Professor at the Department of Electrical and Electronic Engineering (EEE) \& Computer Engineering (CoE) of AIUB. He is also serving as the Head of CoE Department. He is enrolled for $\mathrm{PhD}$ Degree at Universiti Sultan Zainal Abidin, Malaysia. Mr. Zishan is a member of the Institute of Electrical and Electronics Engineers (IEEE) and Institution of Engineers, Bangladesh (IEB). His current research interest includes Wireless Communication, Signal Processing, E-Health System, Telemedicine, Robotics and AI. 
BLANK 